\title{
Assessment of Post Abortion Contraceptive Intention and Associated Factors among Abortion Clients in Gondar Town, North West Ethiopia, 2013
}

\author{
Dejenie Seyoum ${ }^{1}$, Abebaw Gebeyehu ${ }^{2}$, Zemichael Gizaw $^{3, *}$ \\ ${ }^{1}$ Marie Stops International Ethiopia, Gondar branch, Gondar City, Ethiopia \\ ${ }^{2}$ Department of reproductive health, Institute of Public Health, College of Medicine and Health Sciences, Gondar University, Gondar City, \\ Ethiopia \\ ${ }^{3}$ Department of Environmental and Occupational Health and Safety, Institute of Public Health, College of Medicine and Health Sciences, \\ Gondar University, Gondar City, Ethiopia \\ *Corresponding Author: chernetgizaw@yahoo.com
}

Copyright (C) 2014 Horizon Research Publishing All rights reserved.

\begin{abstract}
Unintended pregnancies continue to be a major global tragedy for millions of women and abortion is an issue which affects every country in the world. The World Health Organization estimates that 20 million unsafe abortions take place each year, in which 80,000 maternal deaths occur due to complications. Cross- sectional study was employed to assess post abortion contraceptive intention and associated factors among abortion clients in Gondar town, Northwest Ethiopia 2013. Six hundred thirty study subjects were included in the study by using a simple random sampling technique. The collected data entered and analyzed by using SPSS version 20.0 software packages. The degree of association between independent and dependent variables were assessed using odds ratio with $95 \%$ confidence interval and $\mathrm{p}$-value $\leq 0.05$. The data analyzed using bivariate and multivariate logistic regression. A total of 630 study participants with abortion were assessed. The extent of the post abortion contraceptive intention among 630 clients was (73.5\%). Divorced/widowed women [AOR: 0.277 , 95\% CI : (0.151, 0.508).] was a factor negatively associated with the post abortion contraceptive intention whereas married women [AOR: 1.747, 95\% CI :( 1.046, 2.916)], abortion from unintended pregnancy [AOR: $3.430,95 \%$ CI (1.597, 7.366) and post abortion contraceptive counseling [AOR: $2.627,95 \%$ CI: $(1.366,5.049)]$ were factors positively associated with post abortion contraceptive intention. Generally, the post abortion contraceptive intention found in this study was low as compared with others study. Variables like married women, divorced/widowed, abortion from unintended pregnancy and post abortion contraceptive counseling were significant predictors of the post abortion contraceptive intention.
\end{abstract}

Keywords Post Abortion, Modern Contraceptive, Intention

\section{Introduction}

Globally 180-200 million pregnancies occurred per year. Among them 75 million pregnancies become unwanted and 585,000 Maternal Mortality occur related to pregnancy complication. Among the maternal mortality , $99 \%$ occur in the developing world (1). Globally different factors contribute to this large number of maternal mortality, among them abortion accounts for approximately $13 \%$ of all maternal mortality (2).

Abortion is an issue which affects every country in the world. World Health Organization estimates that 20 million unsafe abortions take place each year, in which 80,000 maternal deaths occur due to complication (3). The unsafe abortion defined by WHO as a procedure for terminating an unintended pregnancy either by persons lacking the necessary skills or in an environment lacking the minimal medical standards, or both(4).

The abortion mortality rate observed in developing and developed countries were 400 and 0.2-1.2 per 100,000 abortions respectively. But this mortality rate became worst in Africa which was 680 per 100,000 abortion cases (5).

Ethiopia, as in many developing countries, pregnancy related complications are believed to be one of the leading causes of death of women among reproductive age groups .It has also high maternal mortality ratio which is 676 per 100,000 live births and low modern family planning coverage which is $27 \%$ among married women (6) .These have a great contribution for unintended pregnancy for sexual active age group in the country. These Untimely and unwanted pregnancies adversely affect the health of women which leads to unsafe abortion which end up in morbidity and mortality among women in her reproductive age.

Ethiopia has a reform of abortion law since 2005 and the penal code was revised to broaden the indications under which abortion is permitted in the country to reduce maternal 
mortality due to unsafe abortion. The revised law permitting to terminate the pregnancy when the pregnancy results from rape or incest, when continuation of the pregnancy endangers the health or life of the Woman or the fetus, in cases of fetal impairment, women with physical or mental disabilities, minors who are physically or psychologically unprepared to raise a child and in cases of grave and imminent danger that can be averted only through immediate pregnancy termination(7).

The research done in Ethiopia in 2008 after the liberalization of abortion law revealed that an estimated 382,000 induced abortions were performed. Among them, 103,000 legal procedures performed in the health facilities. As a result of this the abortion rate was 23 per 1,000 women among the age group of 15-44(8).In another study conducted in Ethiopia, the national case fatality rate 628 per 100,000 among women seeking post abortion care was the most serious complications seen in public hospitals (9). These show that unsafe abortion is a prevalent and serious public health problem among women of reproductive age group in the country.

Ethiopia is one of the country with high maternal mortality ratio and high prevalence of unsafe abortion among reproductive age in the country. These woman health condition become a major concern for the health authorities to liberalize the abortion law in 2005 .This liberalized law is a good opportunity for both the clients and the health institution to avert the incidence of abortion by providing modern contraceptive method after abortion service.

The post abortion contraceptive intention of women has paramount benefit to reduce maternal mortality from unintended pregnancy; in spite of this fact, this component remains one of the weakest parts of post abortion care. Moreover, my knowledge is concerned no study is done in the particular study area especially in Amhara region, Gondar town. So the result of this study will determine the current status of the proportion of women that have intention for modern contraceptive method and associated factors after abortion care. Therefore, the study will be serving as a source of information for stakeholders and partner's to make possible interventions to improve the level of post abortion contraceptive intention in Gondar town.

\section{Objectives of the Study}

\section{General objective}

Assess the post abortion contraceptive intention and associated factors among abortion clients in Gondar town in 2013.

Specific objectives

- To determine the proportion of women s' intention for modern contraceptive methods after abortion care.

- To identify factors associated with the post abortion contraceptive intention.

\section{Methods and Materials}

\section{Study design}

An institution based cross-sectional quantitative study was conducted to assess the post abortion contraceptive intention among abortion clients.

\section{Source population}

All women who came to the Hospital gynecology ward/OPD, health centers and clinical examination room with a diagnosis of abortion and seeking termination of abortion services in Gondar town.

\section{Study population}

All women who came to the Hospital gynecology ward, health centers and clinical examination room with a diagnosis of abortion and seeking termination of abortion services with in July to September.

\section{Inclusion criteria}

Clients who came for both safe abortion and post abortion care services were included in the study.

Exclusion criteria: Those referral cases were excluded from the study.

\section{Sample size}

The Sample size is determined by using single population proportion formula with the following assumption: $\mathbf{z \alpha} / \mathbf{2}=$ (1.96), is standardized normal distribution curve /value for the $95 \%$; proportion of post abortion contraceptive intention which is $83 \%(10)$; the margin of error taken $(0.03)$; Non response rate $10 \%$

$$
n=\frac{\left(\frac{z \alpha}{2}\right)^{2} p q}{W^{2}}
$$

The total sample size for this study was 662 of women seeking abortion care in the study area including $10 \%$ non-response rate.

\section{Sampling procedure}

Among the different health institution from public, private and NGO based clinics found in Gondar town, eleven health institution has provided abortion service and report to the Gondar town health office. Among them, Gondar referral Hospital, Salem medium clinic, MSIE Gondar clinic, FGA-Gondar clinic and poly health centre were selected by a simple random sampling technique using lottery methods.

The sampling allocation was based on the three months average number of client flow for abortion services in each of the health institution. The three months average abortion services for Gondar Hospital was 120 clients, Selam medium clinic 100 clients, poly health centre 65 clients, Gondar MSIE 120 clients and Gondar FGA 70 clients. Proportional allocation for each of the selected institutions using a sample size of 662 was used and the allocated sample for each 
institution became Gondar Hospital 167 clients, MSIE Gondar clinic 167 clients, Selam medium clinic 139 clients, FGA clinic 98 clients and poly health centre 91 clients.

\section{Data collection tools and procedures}

Data were collected using a structured questionnaire. The questionnaire was first prepared in English then translated into the local language (Amharic) and data collected by interviewing clients that fulfill the inclusion criteria.

One supervisor and five data collectors had participated in the data collection process and took two days intensive training with field practice on how to conduct the data collection process to maximize data quality throughout the collection period. The period of data collection took three months in the selected health institution from July 1September 30, 2013.

\section{Variables of the study}

Dependent variable

Post abortion contraceptive intention (Yes/No)

Independent/ explanatory variables

1. Socio-demographic variables: Age, marital status, religion, residential area, women Occupation, women Educational status, family income

2. Previous history: Number of children, previous induced abortion, current contraceptive while pregnant, previous visit of this institution, source of information for FP

3. Present history: Desire of pregnancy, type of institution, method of termination, post abortion FP counseling

\section{Operational definitions}

Post abortion contraceptive intention: a post abortion client's future need of using family planning methods after termination of pregnancy and including those clients who uses family planning immediately after the procedure considered as having the intention for modern family planning method.

Current contraceptive while pregnant: women came for abortion service in spite of taking contraceptive.

Desire of pregnancy: Abortion clients terminate either wanted or unwanted pregnancy.

Post abortion family planning practice: the abortion clients prefer and used modern contraceptive method after the medical or surgical procedure.

\section{Data Processing and Analysis}

The collected data were cleaned and entered into EPI INFO version 2000 statistical software and then transferred to SPSS Windows version 20.0 for further analysis. Frequencies and cross tabulations were used to summarize descriptive statistics of the data using tables and figures that used for data presentation. Bivariate analysis was used primarily to check which variables had association with the dependent variable individually. Variables found to have an association with the dependent variable was then entered into Multiple Logistic regression for controlling the possible effect of confounders and finally the variables which had significant association were identified on the basis of OR, with $95 \% \mathrm{CI}$ and $\mathrm{p}$-value 0.05 to fit into the final regression model.

\section{Ethical Consideration}

Ethical clearance was obtained from the Ethical review board of the University of Gondar. Communication with the head of each health institution and department head was made through formal letter obtained from the University of Gondar. After the purpose and the objective of the study had informed, verbal consent was obtained from each study participant. Participants were informed about they had the right to withdraw from the study at any time if they were not comfortable about the questionnaire. In order to keep confidentiality of any information provided by the study subjects, the data collection procedure was maintained anonymous. The study participants were informed about the purpose of the study, procedure of the study, possible risks and benefits from the study.

\section{Results and Discussion}

\section{Socio-demographic characteristics of the study Participants}

A total of 662 post abortion clients, in the reproductive age group of 15-49 years, were planned and only 630 clients participated in this study with a response rate of $95 \%$. The mean $( \pm \mathrm{SD})$ age of the respondents was $24.12( \pm 5.11)$ years and the majority age group $(41 \%)$ of the respondents were between the age group of 20-24 years. Majority of the respondents were single (57.9\%), urban dwellers (74.3\%), secondary school students (43.7) and had no children before this time (63.3\%) (Table1).

\section{The present history characteristics of respondents}

Majority of the respondents (56.7\%) had mistimed pregnancy. Most of the respondents (92.5\%) got the post abortion contraceptive counselling; among them (90.3\%) understands the benefit of family planning service and the presence of different contraceptive methods (Table 2).

\section{The previous history characteristics of the respondent}

The majority of the respondents $(92.4 \%)$ had sources of information for the different family planning services. Among the different source, $43 \%$ of the respondents got information about family planning from radio. In spite of took contraceptive, one third of the respondent $(29.5 \%)$ had pregnancy specifically due to the inappropriate use of combined oral contraceptive pill and emergency contraceptive pill (Table 3). 
Table 1. Socio demographic characteristics of post abortion clients in Gondar town, 2013

\begin{tabular}{|c|c|c|}
\hline Socio-demographic characteristics & Frequency & Percent \\
\hline \multicolumn{3}{|l|}{ Age } \\
\hline $15-19$ & 107 & 17 \\
\hline $20-24$ & 258 & 41 \\
\hline $25-29$ & 165 & 26.2 \\
\hline $30-34$ & 75 & 11.9 \\
\hline $35-49$ & 25 & 4 \\
\hline \multicolumn{3}{|l|}{ Marital status } \\
\hline Single & 365 & 57.9 \\
\hline Married & 194 & 30.8 \\
\hline Divorced/widowed & 71 & 11.3 \\
\hline \multicolumn{3}{|l|}{ Religion } \\
\hline Orthodox & 524 & 83.2 \\
\hline Muslim & 89 & 14.1 \\
\hline Others* & 17 & 2.7 \\
\hline \multicolumn{3}{|l|}{ Women Education } \\
\hline Primary and below & 181 & 28.7 \\
\hline Secondary & 275 & 43.7 \\
\hline Tertiary & 174 & 27.6 \\
\hline \multicolumn{3}{|l|}{ Residential area } \\
\hline Urban & 468 & 74.3 \\
\hline Rural & 162 & 25.7 \\
\hline \multicolumn{3}{|l|}{ Women Occupation } \\
\hline Student & 209 & 33.2 \\
\hline Government employee & 98 & 15.6 \\
\hline Private employee & 142 & 22.5 \\
\hline Housewife & 115 & 18.3 \\
\hline Jobseeker & 51 & 8.1 \\
\hline Others** & 15 & 2.4 \\
\hline \multicolumn{3}{|l|}{ Family Income assessment } \\
\hline Poor & 198 & 31.4 \\
\hline Medium & 234 & 37.1 \\
\hline Rich & 198 & 31.4 \\
\hline
\end{tabular}


Table 2. Present history characteristics of post abortion clients in Gondar town in 2013

\begin{tabular}{|c|c|c|c|}
\hline present history & characteristics & Frequency & Percent \\
\hline \multicolumn{4}{|c|}{ Desire of pregnancy } \\
\hline & Unwanted & 238 & 37.8 \\
\hline & Wanted & 35 & 5.6 \\
\hline & Mistimed & 357 & 56.7 \\
\hline \multicolumn{4}{|c|}{ Method of Termination } \\
\hline & Pill & 495 & 78.6 \\
\hline & MVA & 119 & 18.9 \\
\hline & Both & 16 & 2.5 \\
\hline \multicolumn{4}{|c|}{ Type of institution } \\
\hline & Public & 240 & 38.1 \\
\hline & Private & 138 & 21.9 \\
\hline & NGO clinics & 252 & 40 \\
\hline \multicolumn{4}{|c|}{ PAFPC } \\
\hline & No & 47 & 7.5 \\
\hline & Yes & 583 & 92.5 \\
\hline \multicolumn{4}{|c|}{ Benefit of FP } \\
\hline & No & 61 & 9.7 \\
\hline & Yes & 569 & 90.3 \\
\hline \multicolumn{4}{|c|}{ Choice of FP } \\
\hline & No & 62 & 9.8 \\
\hline & Yes & 568 & 90.3 \\
\hline \multicolumn{4}{|c|}{ Male as a partner } \\
\hline & No & 496 & 78.7 \\
\hline & Yes & 134 & 21.3 \\
\hline \multicolumn{4}{|c|}{ Side effect of contraceptive } \\
\hline & No & 173 & 27.5 \\
\hline & Yes & 457 & 72.5 \\
\hline \multicolumn{4}{|c|}{ Duration of FP } \\
\hline & No & 157 & 24.9 \\
\hline & Yes & 473 & 75.1 \\
\hline \multicolumn{4}{|c|}{ Presence of medical illness } \\
\hline & No & 487 & 77.3 \\
\hline & Yes & 143 & 22.7 \\
\hline
\end{tabular}


Table 3. previous history characteristics of abortion clients in Gondar town in 2013

\begin{tabular}{|c|c|c|c|}
\hline previous history & characteristics & Frequency & Percent \\
\hline \multicolumn{4}{|c|}{ Number of children } \\
\hline & No children & 399 & 63.3 \\
\hline & One and more children & 231 & 36.7 \\
\hline \multicolumn{4}{|l|}{ Previous abortion } \\
\hline & No & 539 & 85.6 \\
\hline & Yes & 91 & 14.4 \\
\hline \multicolumn{4}{|c|}{ Current contraceptive while pregnant } \\
\hline & No & 444 & 70.5 \\
\hline & Yes & 186 & 29.5 \\
\hline \multicolumn{4}{|c|}{ Previous visit of institution } \\
\hline & No & 471 & 74.8 \\
\hline & Yes & 159 & 25.2 \\
\hline \multicolumn{4}{|c|}{ Who have information on family planning } \\
\hline & No & 48 & 7.6 \\
\hline & Yes & 582 & 92.4 \\
\hline \multicolumn{4}{|c|}{ Type of source of information } \\
\hline \multicolumn{4}{|l|}{ Radio } \\
\hline & No & 359 & 57 \\
\hline & Yes & 271 & 43 \\
\hline \multicolumn{4}{|l|}{ Television } \\
\hline & No & 383 & 60.8 \\
\hline & Yes & 247 & 39.2 \\
\hline \multicolumn{4}{|l|}{ Public health institution } \\
\hline & No & 421 & 66.8 \\
\hline & Yes & 209 & 33.2 \\
\hline \multicolumn{4}{|l|}{ Peer } \\
\hline & No & 422 & 67 \\
\hline & Yes & 208 & 33 \\
\hline \multicolumn{4}{|l|}{ Private health institution } \\
\hline & No & 547 & 86.8 \\
\hline & Yes & 83 & 13.2 \\
\hline \multicolumn{4}{|l|}{ Leaflet } \\
\hline & No & 616 & 97.8 \\
\hline & Yes & 14 & 2.2 \\
\hline
\end{tabular}




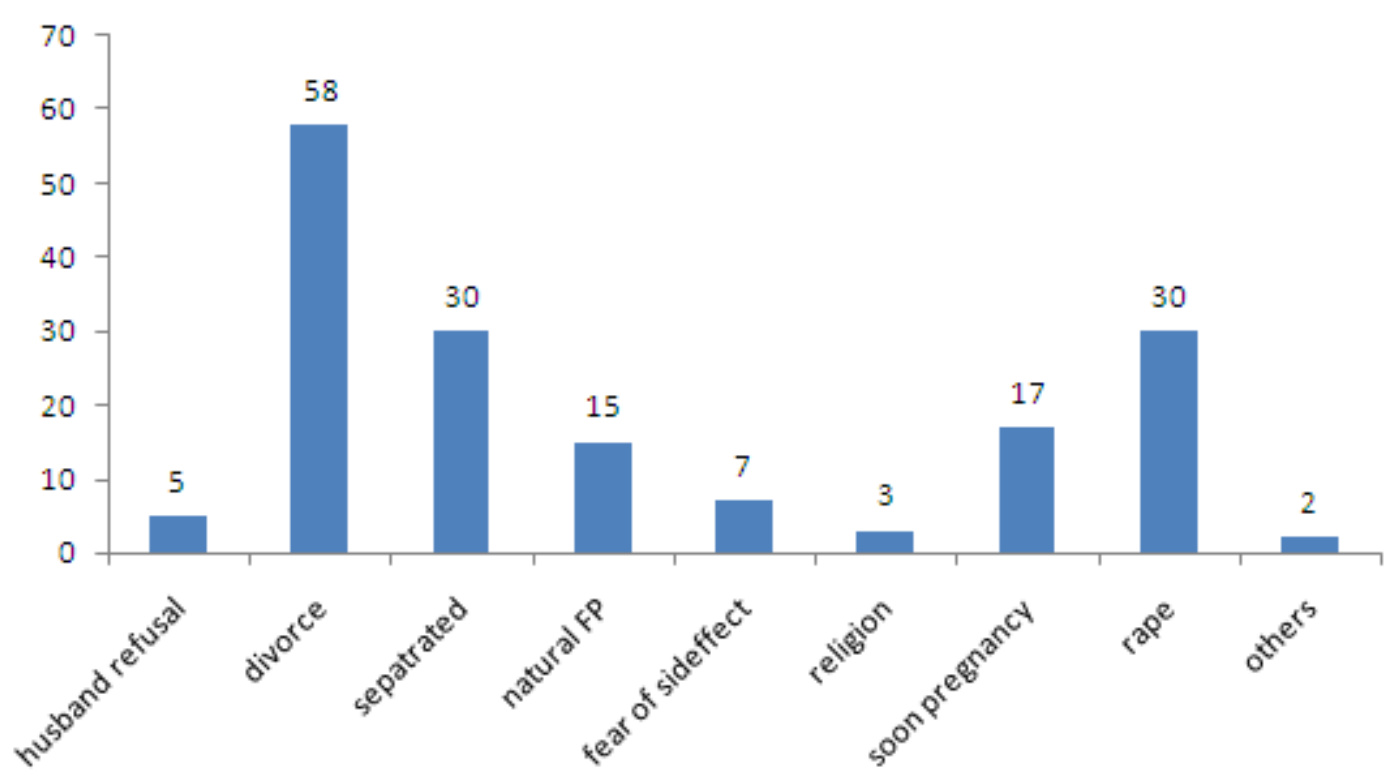

Figure 1. Reason for post abortion clients for not having intention after abortion service in Gondar town in 2013.

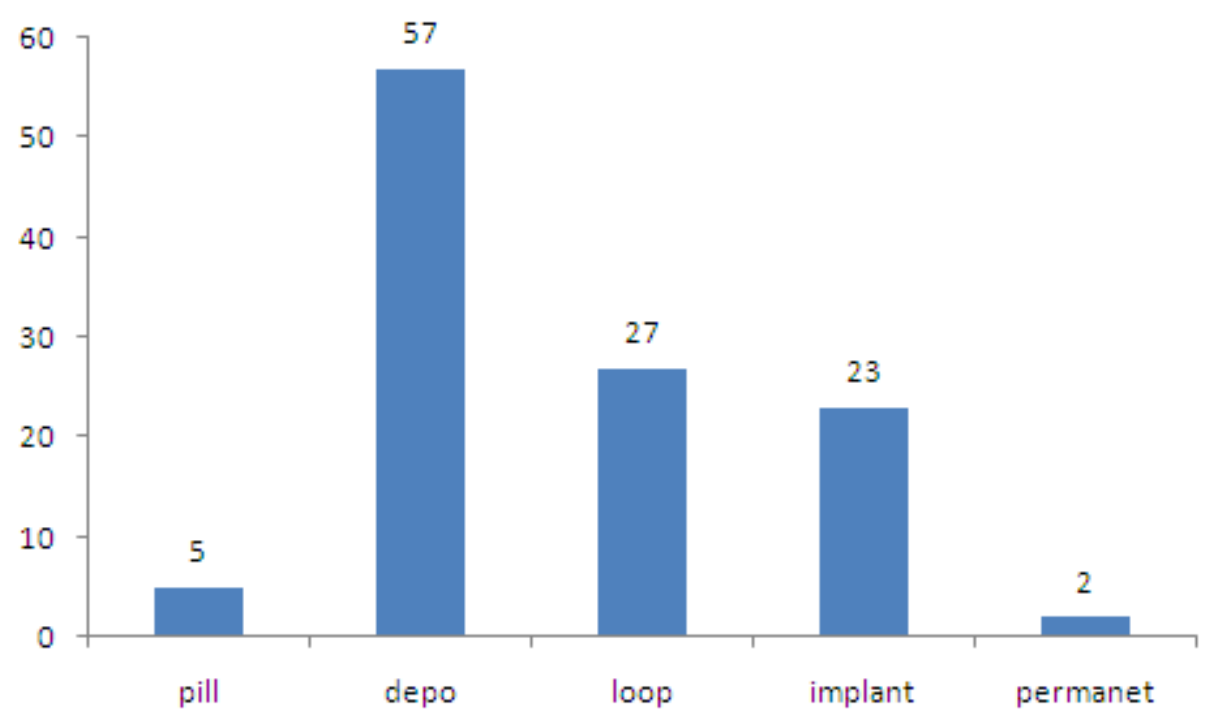

Figure2. Future contraceptive needs of post abortion clients in Gondar town in 2013

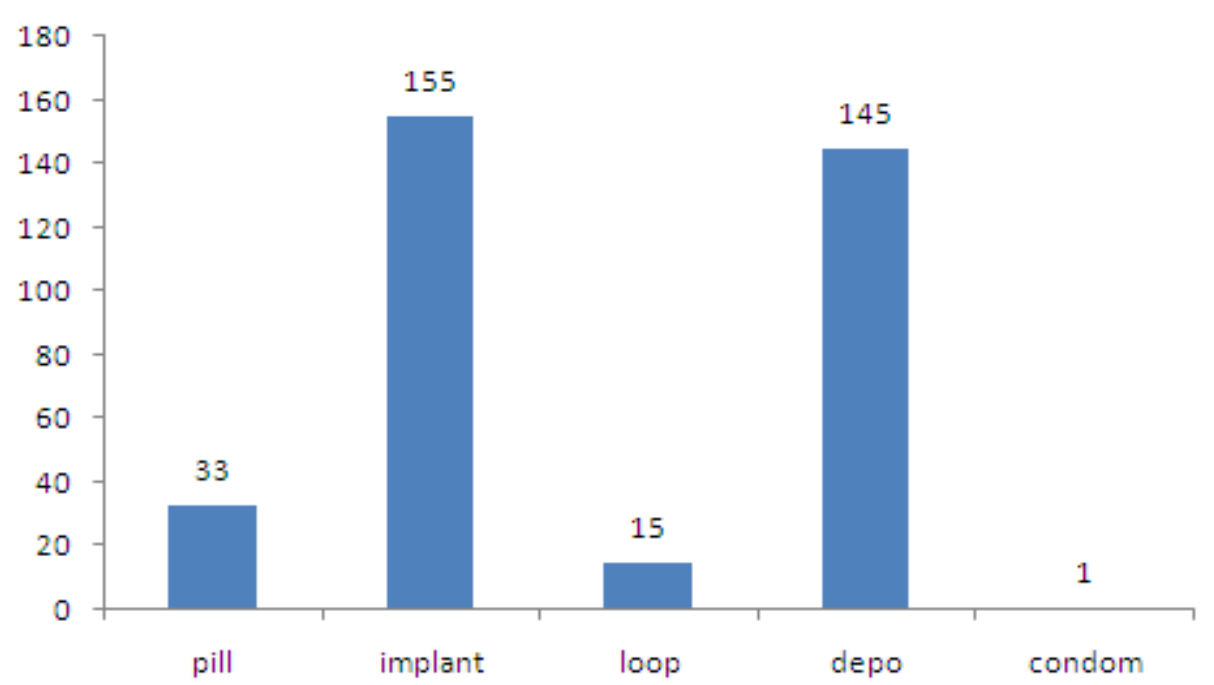

Figure 3. Actual contraceptive users of post abortion clients in Gondar town in 2013 


\section{Post abortion contraceptive intention}

Here after all the post abortion contraceptive intention found in this study was $73.5 \%$ which includes both actual contraceptive users and those who have the need to use contraceptive in the future. On the other hand, those clients who didn't have intention for contraceptive contributed $26.5 \%$. One third of the respondents (26.5\%) had no intention to use post abortion contraceptive. The main reason for not having intention was divorced (34.73\%) and those separated and raped respondents each of them contribute $(17.96 \%)$ for not having intention for contraceptive after abortion service.
More than two third of the respondents 463 (73.5\%) had the intention to use modern contraceptive after abortion service .Among them $114(18.1 \%)$ will have the intention to use contraceptive in the future.

More than two third of the respondent 463 (73.5\%) had the intention to use post abortion family planning contraceptive. Among them 349 (55.4\%) were actual contraceptive users after abortion service.

\section{Factors associated with the post abortion contraceptive intention}

Multivariate analysis showing association of variables with post abortion contraceptive intention

Table 1. Association of variables with post abortion clients on contraceptive intention after abortion service in Gondar town in 2013

\begin{tabular}{|c|c|c|c|c|}
\hline \multirow{2}{*}{ Variable } & \multicolumn{2}{|c|}{ Contraceptive intention } & \multirow[t]{2}{*}{$\operatorname{COR}(95 \% \mathrm{CI})$} & \multirow[t]{2}{*}{$\operatorname{AOR}(95 \% \mathrm{CI})$} \\
\hline & No & Yes & & \\
\hline \multicolumn{5}{|l|}{ Age category } \\
\hline $15-19$ & 24 & 83 & 1 & 1 \\
\hline $20-24$ & 60 & 198 & $0.954(0.557,1.635)$ & $0.986(0.534,1.820)$ \\
\hline $25-29$ & 47 & 118 & $0.726(0.412,1.279)$ & $0.744(0.364,1.523)$ \\
\hline $30-34$ & 28 & 47 & $0.485(0.253,0.932)^{*}$ & $0.564(0.224,1.423)$ \\
\hline $35-49$ & 8 & 17 & $0.614(0.236,1.597)$ & $0.679(0.197,2.340)$ \\
\hline \multicolumn{5}{|l|}{ Marital status } \\
\hline Single & 86 & 279 & 1 & 1 \\
\hline Married & 38 & 156 & $1.265(0.824,1.944)$ & $1.747(1.046,2.916)^{*}$ \\
\hline Divorced /widowed & 43 & 28 & $0.201(0.118,0.342)^{*}$ & $0.277(0.151,0.508)^{*}$ \\
\hline \multicolumn{5}{|l|}{ Religion } \\
\hline Orthodox & 133 & 391 & 1 & 1 \\
\hline Others* & 34 & 72 & $0.720(0.458,1.133)$ & $0.818(0.489,1.368)$ \\
\hline \multicolumn{5}{|l|}{ Education } \\
\hline Below primary & 64 & 117 & 1 & 1 \\
\hline Secondary & 70 & 205 & $1.602(1.065,2.409) *$ & $0.950(0.559,1.614)$ \\
\hline Tertiary & 33 & 141 & $2.337(1.437,3.801)^{*}$ & $1.314(0.677,2.552)$ \\
\hline \multicolumn{5}{|l|}{ Residence } \\
\hline Urban & 115 & 353 & 1 & 1 \\
\hline Rural & 52 & 110 & $0.689(0.466,1.019)$ & $0.824(0.516,1.315)$ \\
\hline \multicolumn{5}{|l|}{ Occupation } \\
\hline Govern. Employee & 23 & 75 & 1 & 1 \\
\hline Private employee & 32 & 110 & $1.054(0.572,1.942)$ & $1.369(0.719,2.604)$ \\
\hline
\end{tabular}




\begin{tabular}{|c|c|c|c|c|}
\hline House wife & 43 & 72 & $0.513(0.282,0.936)^{*}$ & $0.769(0.394,1.503)$ \\
\hline Students & 42 & 167 & $1.219(0.685,2.171)$ & $1.314(0.713,2.423)$ \\
\hline Jobseeker/others & 27 & 39 & $0.443(0.225,0.872)^{*}$ & $0.549(0.269,1.119)$ \\
\hline \multicolumn{5}{|l|}{ institution choice } \\
\hline Public & 74 & 166 & 1 & 1 \\
\hline Private & 40 & 98 & $1.092(0.690,1.728)$ & $0.806(0.487,1.337)$ \\
\hline NGO & 53 & 199 & $1.674(1.113,2.518)^{*}$ & $1.280(0.806,2.031)$ \\
\hline \multicolumn{5}{|l|}{ institution visit } \\
\hline No & 116 & 355 & 1 & 1 \\
\hline Yes & 51 & 108 & $0.692(0.467,1.026)$ & $0.827(0.530,1.290)$ \\
\hline \multicolumn{5}{|l|}{ History of abortion } \\
\hline No & 148 & 391 & 1 & 1 \\
\hline Yes & 19 & 72 & $1.434(0.836,2.461)$ & $1.047(0.556,1.970)$ \\
\hline \multicolumn{5}{|l|}{ Desire of pregnancy } \\
\hline Wanted & 17 & 18 & 1 & 1 \\
\hline Unintended & 150 & 445 & $2.802(1.408,5.576)$ & $3.430(1.597,7.366)^{*}$ \\
\hline \multicolumn{5}{|l|}{ PAFPC } \\
\hline No & 24 & 23 & 1 & 1 \\
\hline Yes & 143 & 440 & $3.211(1.758,5.863)$ & $2.627(1.366,5.049)^{*}$ \\
\hline \multicolumn{5}{|l|}{$\begin{array}{l}\text { Source of } \\
\text { information }\end{array}$} \\
\hline No & 17 & 31 & 1 & 1 \\
\hline Yes & 150 & 432 & $1.579(0.850,2.936)$ & $1.566(0.802,3.058)$ \\
\hline \multicolumn{5}{|l|}{ FP while pregnant } \\
\hline No & 127 & 317 & 1 & 1 \\
\hline Yes & 40 & 146 & $1.462(0.974,2.194)$ & $1.120(0.713,1.760)$ \\
\hline \multicolumn{5}{|l|}{ Wealth index } \\
\hline Poor & 59 & 139 & 1 & 1 \\
\hline Medium & 65 & 169 & $1.104(0.727,1.676)$ & $0.837(0.521,1.343)$ \\
\hline Rich & 43 & 155 & $1.530(0.971,2.411)^{*}$ & $1.195(0.717,1.990)$ \\
\hline \multicolumn{5}{|l|}{ Parity } \\
\hline Nulliparous & 95 & 304 & 1 & 1 \\
\hline Primiparous & 32 & 76 & $0.742(0.463,1.191)$ & $1.055(0.565,1.971)$ \\
\hline Above primiparous & 40 & 83 & $0.648(0.471,1.009)^{*}$ & $1.171(0.593,2.314)$ \\
\hline
\end{tabular}




\section{Discussion}

The level of post abortion contraceptive intention in this study was $73.5 \%$ based on the structured questionnaire. Factors found to be associated with the dependent variable were married, divorced/widowed, desire of pregnancy and post abortion family planning counseling have found to be a significant effect on the outcome variable.

The finding of this study $73.5 \%$ of post abortion contraceptive intention was below the study done in India and Nepal which were $100 \%$ (11) and $83 \%$ (10) respectively. Again the research conducted in Tanzania for post abortion contraceptive $89 \%$ suggests that high rate of contraceptive intention (12). The study conducted in Pakistan for post abortion contraceptive use $72.9 \%$ observed was almost similar finding as this study in 2011(13). But on the contrary, the research done in India in 2012 and the research done in Kenya in 2004 show low contraceptive intention after abortion (30\% and $31 \%$ respectively) than the finding of this study $(14,15)$.

Married have $74.7 \%$ more likely to have post abortion contraceptive intention than being single. But on the contrary the research done in Addis Ababa shows that marital status had not statistically significant association with the odds of using post abortion care services with a modern family planning method (13).

Divorced/widowed women reduced by $72.3 \%$ to have post abortion contraceptive intention than single. On the contrary the research done in Addis Ababa shows that marital status had not statistically significant association with the odds of using post abortion care services with a modern family planning method (13).

Clients got the post abortion contraceptive counseling have 2.63times higher for having contraceptive intention than from who did not get contraceptive counseling. But this finding was a bit lower than the research done in Kenya which stated that Women who received contraceptive counseling were three times more likely to adopt a modern contraceptive method (15).

Abortion of unintended pregnancy has 3.43 times higher for having post abortion contraceptive intention than abortion from wanted pregnancy. This finding was a bit higher than the research done in Kenya which shows that Women who had unplanned pregnancy were three times more likely to adopt a contraceptive method (15).

The post abortion contraceptive practice used in this study was 55.4\%; among them IUCD and permanent family planning contributed $4.3 \%$ and $0 \%$ respectively. But this finding was very low finding than the study conducted in India for IUCD and permanent family planning were $51.5 \%$ and $24.7 \%$ respectively (11).

The future contraceptive need found in this study was $18.1 \%$. Among them IUCD and permanent were $23.68 \%$ and $1.7 \%$ respectively. This IUCD finding was in line with the study conducted at Karnataka which was $22.7 \%$ but the permanent method found in this study was very low $(22.7 \%)$ than again the study conducted at Karnataka (16).

\section{Conclusions}

Based on the findings of this study we can conclude that generally the post abortion contraceptive intention was low as compared to other study. Factors associated with the dependent variable was divorced/widowed negatively associated with post abortion contraceptive intention and married women, desire of pregnancy and post abortion family planning counseling were positively associated with the post abortion contraceptive intention.

\section{REFERENCES}

[1] Hani wahib Fawzi MPH M. Maternal mortality reduction. sudanese journal of public health October 2006;1(4).

[2] M. B. Making Abortions Safe: A Matter of Good Public Health Policy and Practice. Bulletin of the World Health Organization. 2000;78(5):580-88.

[3] Wada T. ABORTION LAW IN ETHIOPIA: A Comparative Perspective. Jan 2008;2:1-5.

[4] WHO. Communicating Family Planning in Reproductive Health. 1997.

[5] J Ravindran F. Unwanted Pregnancy - Medical and Ethical Dimensions. Medical Journal of Malaysia March 2003; 58: 23-35.

[6] Agency Cs. Ethiopia Demographic and Health Survey (DHS). 2011.

[7] Ethiopia potFDRo. criminal code of the Federal Democratic Republic of Ethiopia may 2005.

[8] Susheela Singh TF, Hailemichael Gebreselassie , Ahmed Abdella, YirguGebrehiwot, SolomonKumbi and Suzette Audam The Estimated Incidence of Induced Abortion In Ethiopia. 2008;36(1):16-25.

[9] Hailemichael Gebreselassie TF, Susheela Singh, Ahmed Abdella, YirguGebrehiwot, Solomon Tesfaye, TakeleGeressu and SolomonKumbi; ; Volume ,Number 1, 7. Caring for Women with Abortion Complications In Ethiopia :National Estimates and Future Implications. March 2010;36.

[10] Khanal V JC, Neupane D, Karkee R. . Practices and Perceptions on Contraception Acceptance among Clients Availing Safe Abortion Services in Nepal. JUL- SEP 2011;9(35).

[11] UMASHANKAR K.M DMN, JAYANTA KUMAR D.E, KALAK, ABED GULAB

NAGURE,RAMADEVI . .Survey of the Attitude to, the Knowledge and the Practice of Contraception and Medical Abortion in Women Who Attended a Family Planning Clinic.7(3):493-5.

[12] Vibeke Rasch FYaSM. Medium and long-term adherence to post abortion contraception among women having experienced unsafe abortion in Dar es Salaam, Tanzania. 31 July 2008.

[13] ArSyed Khurram Azmat WH, Muhammad Ishaque, Ghulam Mustafa, and Aftab Ahmed Post-abortion care family 
planning use in Pakistan. 2012;2.

[14] Zavier AJ PS. Postabortion contraceptive use and method continuation in India. 2012 118(1):65-70.

[15] Onyango M. A. OM, Barger M. Predictive Factors for Uptake of Post-Abortion Care in Western Kenya. African Journal of
Midwifery and Women's Health. 2010 Jul;4(3):115-20.

[16] Parvati V.Bhat AP, Pratap Kumar and Sreekumaran Nair. Contraceptive knowledge, practice and acceptance among women seeking termination of pregnancyat a secondary level hospital in southern karnataka. 2008;31(3):157-62. 